\title{
Meibomian Gland
}

National Cancer Institute

\section{Source}

National Cancer Institute. Meibomian Gland. NCI Thesaurus. Code C33075.

A sebaceous gland in the eyelid that produces a specific type of sebum. 